\title{
Neural dynamics of error processing in medial frontal cortex
}

\author{
Rogier B. Mars, ${ }^{\text {a,b,* }}$ Michael G.H. Coles, ${ }^{\mathrm{a}}$ Meike J. Grol, ${ }^{\mathrm{a}, \mathrm{c}}$ Clay B. Holroyd, ${ }^{\mathrm{d}}$ \\ Sander Nieuwenhuis, ${ }^{\mathrm{e}}$ Wouter Hulstijn, ${ }^{\mathrm{b}}$ and Ivan Toni ${ }^{\mathrm{a}, \mathrm{b}}$ \\ ${ }^{a}$ F.C. Donders Centre for Cognitive Neuroimaging, P.O. Box 9101, NL-6500 HB Nijmegen, The Netherlands \\ ${ }^{\mathrm{b}}$ Nijmegen Institute for Cognition and Information, Radboud University, P.O. Box 9104, NL-6500 HE Nijmegen, The Netherlands \\ ${ }^{\mathrm{c}}$ Helmholz Institute, P.O. Box 80125, NL-3508 TC Utrecht, The Netherlands \\ ${ }^{\mathrm{d}}$ Department of Psychology, University of Victoria, P.O. Box 3050 STN CSC, Victoria, BC, Canada V8W $3 P 5$ \\ 'Department of Cognitive Psychology, Vrije Universiteit, Van der Boechorststraat 1, 1081 BT Amsterdam, The Netherlands
}

Received 12 April 2005; revised 1 June 2005; accepted 10 June 2005

Available online 1 August 2005

\begin{abstract}
Adaptive behavior requires an organism to evaluate the outcome of its actions, such that future behavior can be adjusted accordingly and the appropriate response selected. During associative learning, the time at which such evaluative information is available changes as learning progresses, from the delivery of performance feedback early in learning to the execution of the response itself during learned performance. Here, we report a learning-dependent shift in the timing of activation in the rostral cingulate zone of the anterior cingulate cortex from external error feedback to internal error detection. This pattern of activity is seen only in the anterior cingulate, not in the presupplementary motor area. The dynamics of these reciprocal changes are consistent with the claim that the rostral cingulate zone is involved in response selection on the basis of the expected outcome of an action. Specifically, these data illustrate how the anterior cingulate receives evaluative information, indicating that an action has not produced the desired result.
\end{abstract}

(C) 2005 Elsevier Inc. All rights reserved.

Keywords: Error processing; Motor learning; Cognitive control; ACC; Cingulate cortex; fMRI

\section{Introduction}

To survive in changing environments, an organism must be able to adapt its behavior to the situation at hand. This flexibility can be achieved by evaluating response outcomes and adjusting behavior accordingly (Dickinson, 1985). In this regard, error signals provide important evaluative information, since they indicate that a behavior was inadequate given the current context and that, in future, a different response needs to be selected (Holroyd and Coles, 2002).

\footnotetext{
* Corresponding author. F.C. Donders Centre for Cognitive Neuroimaging, P.O. Box 9101, NL-6500 HB Nijmegen, The Netherlands. Fax: +31243610989.

E-mail address: rogier.mars@fcdonders.ru.nl (R.B. Mars).

Available online on ScienceDirect (www.sciencedirect.com).
}

Existing data on the neural substrates of action selection indicate that the medial frontal cortex plays a crucial role in selecting actions on the basis of their outcomes (Matsumoto and Tanaka, 2004) and subsequent monitoring of response outcomes (Holroyd et al., 2004a; Ridderinkhof et al., 2004). Rather than attributing a single role to this vast cortical expanse, recent studies have started to associate different functions to the different anatomical structures that lay within the medial frontal cortex (Picard and Strick, 2001; Rushworth et al., 2004). In this context, an anterior portion of the cingulate cortex, the rostral cingulate zone anterior (RCZa), has been specifically associated with processing of error information and selecting appropriate behavioral adjustments (Holroyd and Coles, 2002; Rushworth et al., 2004; Fiehler et al., 2004).

These inferences on the neural bases of error processing have been obtained in the context of a "static" experimental environment, in which the organism knows the behavior that is appropriate for the current situation. Thus, a given response can be evaluated immediately against an internal representation of the correct stimulus-response relationship. Should the response be incorrect, error information is available from an internal error-detection process at the time of the response (Gehring et al., 1993; Holroyd et al., 2005). However, in a novel environment, with as yet unknown stimulus-response associations, error information is not available until the delivery of external performance feedback. This implies that, during the learning of stimulus-response associations by trial and error, the time at which error information is available will change. Prior to learning, error information will not be available until external performance feedback is delivered, but after learning, error information will be available earlier from internal sources at the time of the response itself. Thus, a neural structure that adjusts behavior as a function of the evaluation of response outcomes should dynamically shift its responsivity as a function of learning, from external sources provided by error feedback to internal sources associated with the error response itself. We predicted that, following error feedback, activity in the anterior 


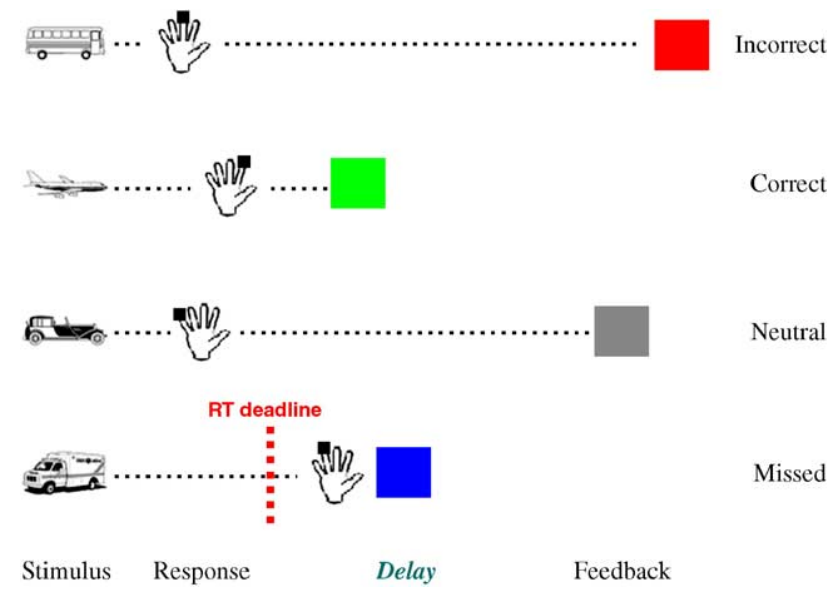

Fig. 1. Task setup. Participants had to learn, by trial and error, arbitrary associations between visual stimuli and motor responses. After a variable delay, visual feedback (red/green square) was provided, indicating correct and incorrect responses. On 50\% of the trials, feedback consisted of a noninformative gray square. When responses occurred after the reaction time deadline $(750 \mathrm{~ms})$, immediate feedback (blue square) was provided.

cingulate cortex would decrease as learning proceeds; conversely, following an erroneous response, activity in the anterior cingulate would increase as learning proceeds. These predictions can be derived from a neuro-computational model (Holroyd and Coles, 2002) that formally describes the relationship between neural systems involved in outcome evaluation with those involved in action selection.

To test these predictions, we asked human subjects to learn arbitrary visuomotor mappings (Wise and Murray, 2000; Toni et al., 2001), using performance feedback, while measuring their cerebral activity using functional magnetic resonance imaging (fMRI). Participants were presented with line drawings, each of which was associated with pressing one of four response buttons (Fig. 1). We manipulated the degree of learning achieved during the scanning session by varying the number of times a given visuomotor mapping was presented. For one condition (High Learning, HL), four distinct visuomotor mappings were presented 36 times each over the course of the scanning session, enabling the subject to fully learn the visuomotor associations. For a control condition (Low Learning, LL), 24 different mappings were presented 6 times each. A reaction time (RT) deadline ensured that participants made errors, even during learned performance. Crucially, by varying the delay between response and feedback, and by introducing neutral feedback on some of the trials, we were able to dissociate the hemodynamic responses elicited by response and feedback (see Experimental timing).

\section{Materials and methods}

\section{Subjects}

We studied eight right-handed male volunteers (mean age = 30.4 years, $\mathrm{SD}=13.4$ ) with normal or corrected-to-normal vision after obtaining informed consent according to institutional guidelines of the local ethics committee (CMO region ArnhemNijmegen, Netherlands). They were paid $€ 10$ per hour for their participation. Imaging data from 5 additional subjects were discarded, since these subjects either failed to learn the appropriate stimulus-response mappings adequately ( 2 subjects, less than $50 \%$ correct on post-scanning forced-choice recall task) or performed without any errors during the last part of the scanning session, indicating that the RT deadline was not tight enough for these subjects (3 subjects).

\section{Experimental setup}

Subjects lay supine in the scanner. Head movements were minimized by an adjustable padded head holder. Visual stimuli (visual angle of approximately $6^{\circ}$ ) were projected onto a mirror above the subjects' heads. Motor responses were recorded via an MR-compatible keypad (MRI Devices, Waukesha, WI), positioned on the right side of the subject's abdomen. Stimulus presentation and response collection were controlled by a PC running Presentation 0.51 (Neurobehavioral Systems, San Francisco, CA).

\section{Behavioral procedure}

Participants were asked to try to learn arbitrary associations between visual stimuli (black and white drawings of cars, airplanes, boats, etc.) and motor responses (pressing of one of four buttons with the fingers of the right hand) by trial-and-error using performance feedback (Fig. 1). We manipulated the degree of learning achieved during the experimental session by varying the number of times a visuomotor mapping was presented. For one condition (High Learning, HL), four distinct visuomotor mappings were presented 36 times each over the course of the scanning session, while for a control condition (Low Learning, LL), 24 different mappings were presented 6 times each. Trials enabling learning (HL) were pseudo-randomly intermixed and matched in number with trials in which learning was less likely to occur (LL). Participants received either performance feedback (green or red square) or neutral feedback (gray square, see Experimental timing) after each response, with a variable delay between these two events. To encourage error commission even during learned performance, a stringent reaction time deadline of $750 \mathrm{~ms}$ was enforced. When subjects responded after this deadline, immediate feedback (blue square) was provided and the trial ended. Subjects were instructed to try to avoid this at all costs. Subjects practiced the task in the scanner for 50 trials using a different stimulus set before the experimental session.

Following the scanning session, participants performed a forced choice recall test, in which all stimuli of the HL condition and a subset $(50 \%)$ of the stimuli of the LL condition were presented 7 times each, randomly intermixed. Subjects were required to press the button corresponding to each stimulus, as during the scanning session. However, during the recall test, there was no reaction time deadline and no feedback was given, to allow for a reliable assessment of the learning of the stimulusresponse mappings.

\section{Imaging procedures}

Images were acquired using a $1.5 \mathrm{~T}$ Sonata scanner (Siemens, Erlangen, Germany). BOLD sensitive functional images were acquired using a single shot gradient EPI sequence (TR/TE $2.2 \mathrm{~s}$ / $40 \mathrm{~ms}, 28$ transversal slices, interleaved acquisition, voxel size $3.5 \times$ $3.5 \times 3.5 \mathrm{~mm})$. Following the experimental session, structural images were acquired using an MP-RAGE sequence (TR/TE/TI $2250 \mathrm{~ms} / 3.93 \mathrm{~ms} / 850 \mathrm{~ms}$, voxel size $1 \times 1 \times 1 \mathrm{~mm}$ ). 


\section{Experimental timing}

Our design was aimed at dissociating response- and feedbackrelated neurovascular activities despite their temporal proximity. We achieved this by using an event-related fMRI design that has proven effective in dissociating transient responses time-locked to sensory and motor events (Thoenissen et al., 2002; Toni et al., 1999).

We introduced a variable delay between response and feedback (3.9-5.2 s, uniform distribution) and between the trials (1.3-13.5 s). Also, we introduced neutral feedback on approximately half of the trials, to decorrelate the stimulus/response and feedback regressors. Furthermore, before actual scanning, we ran simulations in order to optimize the range and order of delay lengths, inter-trial intervals, and neutral feedback stimuli and to minimize correlations between the regressors describing the expected BOLD signal to response and feedback events (Friston et al., 1999). Following the scanning session, we verified the ability of our design to dissociate response and feedback-related activity by examining the evoked hemodynamic responses in V1 and M1. As expected, we found reliable BOLD responses to both the stimulus response epoch and the feedback epoch in V1, but only responserelated activation in M1 (data not shown).

\section{Data analysis}

Imaging data were analyzed using SPM2 (www.fil.ion.ucl.ac. $\mathrm{uk} / \mathrm{spm} /$ ). The first five volumes of each participant's data set were discarded to allow for T1 equilibration. Prior to analysis, data were spatially realigned and corrected for differences in slice time acquisition using the middle slice in time as reference. Each participant's structural image was coregistered to the first of the functional images. Images were then normalized onto the ICBM template (http://www.loni.ucla.edu/ICBM/) using linear transformations only. Finally, data were spatially smoothed using an isotropic $6 \mathrm{~mm}$ FWHM Gaussian kernel.

Using standard multiple regression procedures (Friston et al., 1995), we partitioned the sources of experimental variance in the fMRI time series into main effects of Condition (High Learning or Low Learning), Epoch (activity time-locked either to the response or to feedback presentation), and Outcome (correct or incorrect for response-related data; correct, incorrect, or neutral for feedbackrelated data). Model regressors were convolved with a canonical hemodynamic response function (Friston et al., 1998). Learningdependent modulations of activity were modeled as first and second order parametric effects of time on the model regressors. Confounding factors such as trials with late responses, corrective responses, head-related movements, and trial-by-trial variations in RT were also accounted for and included in the model.

In this paper, we focus our analysis on the rostral cingulate zone anterior $(\mathrm{RCZa})$, a portion of the anterior cingulate cortex which has previously been associated with response errors (Ullsperger and Von Cramon, 2001), negative feedback (Ullsperger and Von Cramon, 2003), and reductions in reward leading to behavioral adjustments (Bush et al., 2002). This area is suggested to correspond to the monkey rostral cingulate motor area (Picard and Strick, 1996) and is situated in what Bush et al. (2000) described as the 'cognitive' division of the anterior cingulate cortex. We also consider a neighboring portion of the superior frontal gyrus, namely the pre-supplementary motor area (preSMA), given its reported role in performance monitoring and

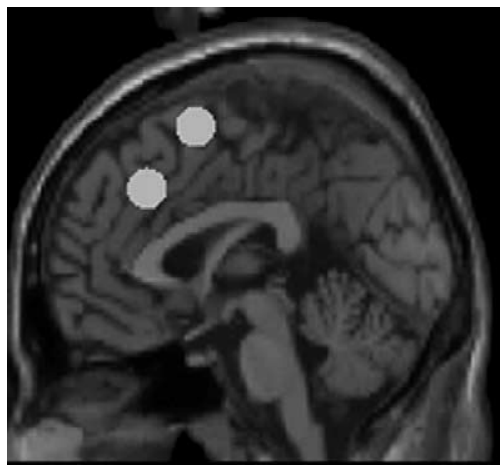

Fig. 2. Anatomical locations of the regions of interest used in the random effect analysis, displayed on the SPM2 canonical single subject T1 image. A spherical region of interest was placed in each hemisphere in each of the anatomically defined structures.

action selection (Fiehler et al., 2004; Rushworth et al., 2004; Shima et al., 1996; Ullsperger and Von Cramon, 2001). For each hemisphere, we created two objectively defined spherical volumes of interest (VOIs, Fig. 2), centered in the 'arm' regions reported by Picard and Strick (1996), and with a radius of $8 \mathrm{~mm}$. The VOIs covering the RCZa were centered at $\pm 8,30,32$; the VOIs covering the pre-SMA were centered at $\pm 8,10,55$, according to the stereotactic coordinates of Talairach and Tournoux (1988) used in the maps of Picard and Strick (1996). These coordinates were converted into the MNI coordinates used by SPM2 using tal2mni (Matthew Brett, http://www.mrc-cbu.cam.ac.uk/Imaging/Common/ downloads/MNI2tal/tal2mni.m).

The statistical significance of the estimated evoked hemodynamic responses was assessed using $t$ statistics in the context of a multiple regression analysis (Poline et al., 2004). Contrasts of the parameter estimates for the Condition $\times$ Time interactions during the incorrect trials were calculated, and entered into a paired $t$ test, treating subjects as a random variable (Holmes and Friston, 1998). The statistical threshold was set at a value of $P<0.05$, corrected for multiple comparisons according to the False Discovery Rate (Genovese et al., 2002) over each of the specified VOIs. To correct for false positives due to the use of multiple VOIs, we applied a further Bonferroni correction to the resulting $P$ values.

In this study, we were interested in assessing differential modulation of time-related signal changes time-locked to feedback or response events during performance of incorrect trials in the HL condition. Accordingly, linear time-dependent increases in activity during the response Epoch on incorrect trials were compared with the corresponding effect during the feedback Epoch (incorrect trials only). Furthermore, to isolate genuine learning-related changes rather than mere time-related effects, we required the Condition $\times$ Time interaction to be stronger in the HL than in the LL condition. This constrain was imposed by selecting voxels in which the Condition $\times$ Time interaction for the response epoch was stronger in the HL than in the LL condition (inclusive mask thresholded at $P<0.05$ uncorrected).

Within the regions identified by our analysis, we calculated the effect sizes for the main and time-related effects, using the ratio of the relevant parameter estimate onto its standard error (Maxwell and Delany, 1990). This allowed us to assess the specificity of the region's activity to errors as compared to correct trials and the presence of main effects of response and feedback.

For analysis of the behavioral data acquired during the scanning session, RT and error rates were each considered as 
dependent variables in a two-way analysis of variance, with factors Condition (2 levels, HL and LL) and Time (8 levels). After removal of missed trials, the RT time series of each participant was divided into eight equal blocks, providing eight levels for the Time factor.

\section{Results}

\section{Behavioral data}

Behavioral data indicated that our design was successful in manipulating the degree of learning achieved by the participants during the scanning session. Participants learned the stimulusresponse mappings at a faster rate in the High Learning condition than in the Low Learning condition (Condition $\times$ Time interaction on Error Rate: $F_{(7,49)}=3.2, P=0.035$, Fig. 3a). Although participants never reached error-free performance during the scanning session in either condition (because of the RT deadline), a post-scanning forced choice recall test indicated that more associations were learned in the High Learning condition (HL: 91\%, LL: $\left.43 \% ; t_{(7)}=12.1, P<0.001\right)$.

RTs on correct trials (Fig. 3b) did not differ between the two conditions (main effect of Condition: $F_{(1,7)}=0.446$, n.s.). Over the course of the scanning session, RT increased to approach the RT cut-off (main effect of Time: $F_{(7,49)}=5.493, P=0.008$ ), but at a similar rate across conditions (Condition $\times$ Time interaction: $F_{(7,49)}=1.514$, n.s.). The number of missed responses did not differ across conditions (HL: $19.7 \%$ [SD = 15.2]; LL: 18.4\% [SD = $12.3] ; t_{(7)}=0.908$, n.s.).

\section{Imaging data}

We isolated BOLD signals satisfying our criteria by testing, in each of the ROIs, for time-dependent response-related increases and feedback-related decreases in activity during error trials. In addition, to distinguish genuine learning-related changes from mere time-related effects, we required this interaction to be stronger in the High Learning condition than in the Low Learning condition.

Our VOI analyses identified a region within the rostral cingulate zone anterior ( $\mathrm{RCZa}$, Table 1) which showed learningrelated changes in activation elicited by incorrect responses and
Table 1

Anatomical specification, MNI coordinates of significant clusters $(P<0.5$ corrected for multiple comparisons), and $Z$ values of clusters yielded by the contrast testing for decreasing time-related changes in BOLD signal at the moment of negative performance feedback, and increasing time-related changes in BOLD signal at the moment of the erroneous response

\begin{tabular}{lrrll}
\hline Anatomical region & \multicolumn{3}{c}{ MNI coordinates } & $Z$ \\
\cline { 2 - 4 } & \multicolumn{1}{c}{$x$} & $y$ & $z$ & \\
\hline Pre-SMA & -8 & 12 & 64 & $2.80^{1}$ \\
& 2 & 4 & 60 & $2.64^{1}$ \\
RCZa & 14 & 10 & 60 & $3.37^{1}$ \\
\hline$Z$
\end{tabular}

$Z$ values marked with ${ }^{1}$ did not survive Bonferroni correction for testing of multiple VOIs.

negative performance feedback. As illustrated in Fig. 4, this region showed greater feedback-related error activation during initial learning. During learning, this feedback-related activation decreased, while the response-related error signal showed a reciprocal increase (Fig. 4c).

To further characterize the activity evoked in this region, we calculated effect sizes for each main and time-related effect, normalizing the relevant parameter estimate of the multiple regression onto its standard error (see Materials and methods). The reciprocity of the dynamic modulation of activity in this cluster is indicated by the presence of significant learning-related effects, but no overall effects of response or feedback. The RCZa showed no significant activity on correct trials (Fig. 4d), indicating that activity in this region was specifically related to error processing. Furthermore, the time-related changes in activation on incorrect trials were stronger in the High Learning (feedback: -3.17 ; response: 2.60 , Fig. 4b) as compared to the Low Learning (feedback: -2.70 ; response: 0.15 ) condition, providing evidence that these changes are not simply due to time-related effects (e.g., fatigue, habituation, sensitization), but are genuinely learningrelated.

There were further clusters of activity in the pre-SMA VOI, although they did not survive the additional Bonferroni correction for multiple VOIs (Table 1). This region showed a clear modulation of activity as a function of learning, as illustrated in Fig. 5, for both response (effect size: 4.20) and feedback (effect size: -3.76), and these modulations were not as strong in the LL condition (feedback: -0.04 ; response: 1.93 ).
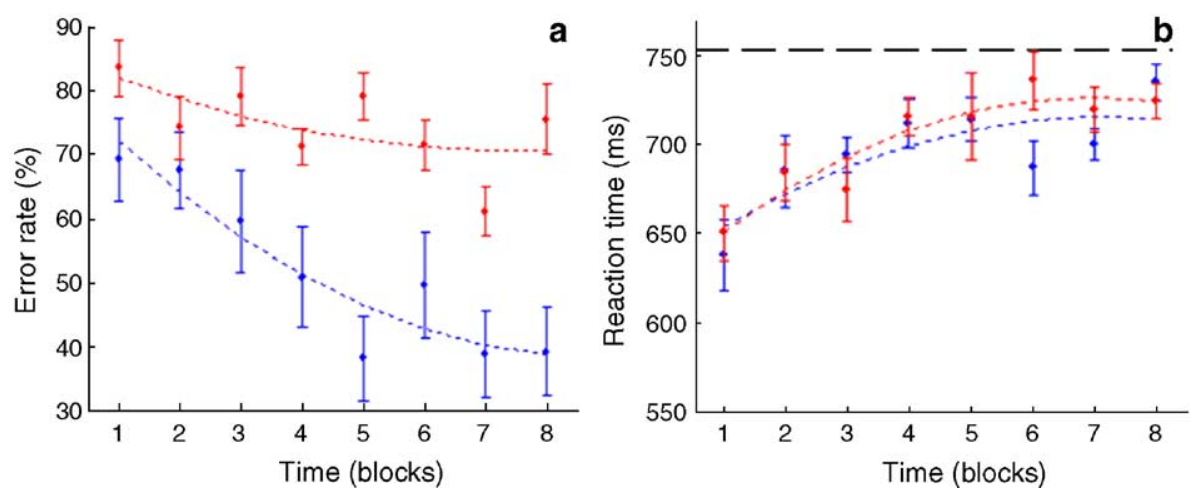

Fig. 3. Behavioral results. Error rates (a) and RT on correct trials (b) for the High Learning (blue) and Low Learning (red) conditions obtained during the scanning session. Curves are fitted first and second order polynomials; error bars indicate \pm SEM. It can be seen that subjects learned the stimulus-response mappings at a faster rate in the High Learning condition than in the Low Learning condition (panel a). RT did not differ between the two conditions, approaching the RT cut-off (dashed horizontal line) at a similar rate (panel b). 

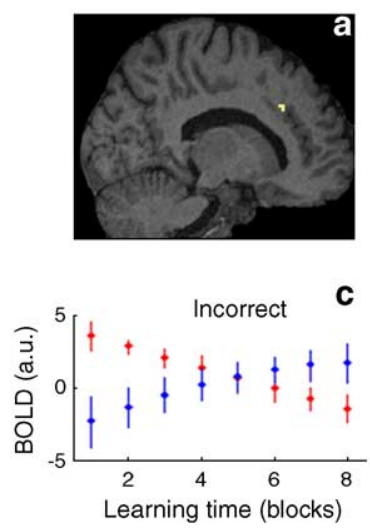
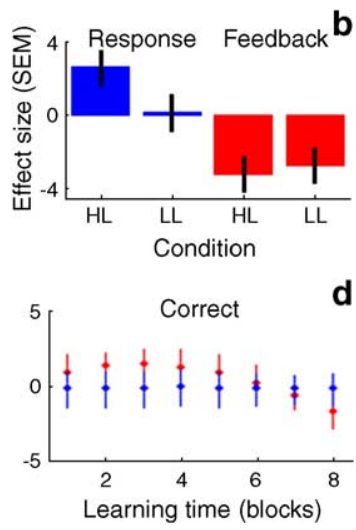

Fig. 4. Imaging results. Anatomical localization, peak BOLD signal development during learning for both incorrect and correct trials (High Learning condition), and effect sizes for time-related modulation in BOLD response for the RCZa (top row, peak coordinates: 14, 28, 32). (a) $\operatorname{SPM}\{Z\}$ (threshold $P<0.05$ corrected) superimposed on normalized anatomical sagittal sections of one participant. (b) Effect sizes (in SEM units) for the time-related changes in BOLD response in both the High Learning (HL) and Low Learning (LL) conditions, indicating stronger modulations of activity in the High Learning condition. (c,d) Peak BOLD signal (in arbitrary units, \pm SEM) over the course of learning, following response (blue) and feedback (red) for incorrect (c) and correct trials (d). For display purposes, the fMRI time series of each subject were subdivided into eight blocks of equal length. The actual statistical model of the fMRI data considered time as a continuous parametric effect (see Materials and methods). It can be seen that error feedback-related activation decreases as learning proceeds, while error response-related activation increases, and these effects are reciprocal.

Crucially, this region did not show the same reciprocity of effects seen in the anterior cingulate clusters, as indicated by a significant main effect of response (effect size: 6.74). Furthermore, the pre-SMA revealed response-related activity during correct trials (effect size: 4.55), an indication that this region is not exclusively driven by error signals.

\section{Discussion}

The present data indicate that, over the course of learning a set of arbitrary visuomotor mappings, a region along the cingulate sulcus (RCZa) shifts its responsiveness to different sources of error information as a function of learning. Error feedback-related activation decreases as learning proceeds, while error responserelated activation increases, and these effects are reciprocal (Fig. 4). These results show not only that the anterior cingulate cortex responds to both internal (Carter et al., 1998; Garavan et al., 2002;
Ullsperger and Von Cramon, 2001) and external (Holroyd et al., 2004b; Ullsperger and Von Cramon, 2003) sources of error information, but also that this cingulate region responds to the earliest source of error information available.

Furthermore, the present data argue against a unique cognitive contribution of the vast expanse of cerebral cortex labeled 'medial frontal cortex', confirming and detailing the functional heterogeneity of different anatomical portions of this region (Nachev et al., 2005; Rushworth et al., 2004). While the RCZa is activated in response to the first signal that an error has occurred, independent of the source of this information, pre-SMA shows response-related effects over and above learning-dependent modulations of activity on both correct and incorrect trials. These findings are consistent with the results of Akkal et al. (2002), showing that CMAr neurons are more likely to be modulated by performance feedback than preSMA neurons. This suggests that pre-SMA might be closer to motor aspects of the learning process than to the generation of error-related behavioral adjustments.

It could be argued that the differential time-related effects seen in the RCZa for the High Learning and Low Learning conditions reflect the putative role of the anterior cingulate region in controlling arousal (Critchley et al., 2003). However, our behavioral data and post-scanning forced choice recall test indicate that subjects learned the stimulus-response mappings in both learning conditions, although to a different extent (Fig. 3a). This result implies that subjects were evaluating stimuli and feedback during both Low and High Learning trials, although the rapid turnover of stimuli-response mappings in the former condition prevented them from learning as effectively as during the latter condition. Moreover, the two experimental conditions evoked overlapping reaction times profiles (Fig. 3b). These behavioral results are not immediately compatible with different arousal levels evoked by the High and Low Learning conditions.

Recently, Walton et al. (2004) have shown that the RCZa can be active not only on incorrect trials, but also on correct trials, provided that these trials convey behaviorally relevant information. In the current learning task, it is possible that the first correct trial associated with a specific mapping might have evoked ACC activity. Unfortunately, the current study was not designed to address this particular issue and we lack an adequate number of "first correct" trials to be able to provide a reliable estimate of ACC activity under these circumstances. This issue remains open for further investigation.

In this study, we have focused our search on RCZa on the basis of the role played by CMAr [the equivalent of RCZa in the macaque (Picard and Strick, 1996)] in reward-based action selection and evaluation (Shima and Tanji, 1998). Although recent meta-analyses (Ridderinkhof et al., 2004; Ullsperger and Von Cramon, 2004) have reported that error processing within the
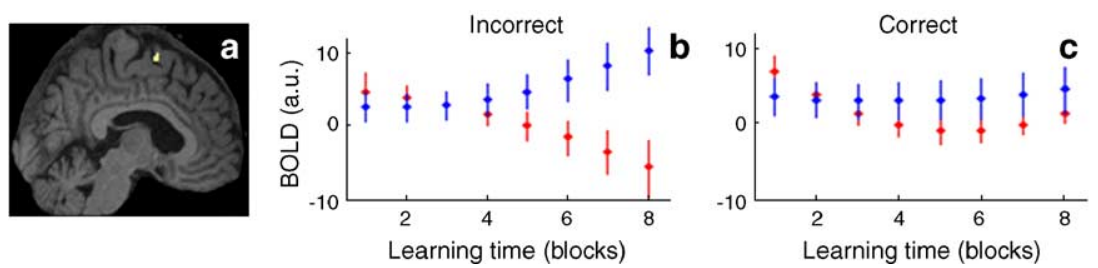

Fig. 5. Imaging results. Anatomical localization and peak BOLD signal development during learning for both incorrect and correct trials (HL condition) for the pre-SMA (peak coordinates: $2,4,60$ ). It can be seen that pre-SMA shows a response-related activation over and above the learning-related modulations of activation, both on correct and incorrect trials. Color conventions as in Fig. 4. 
anterior cingulate may encompass both RCZa and RCZp, the armsubfields of these two areas are structurally and hodologically different (Picard and Strick, 1996), i.e. they are meant to have different functional properties. Indeed, an analysis on the errorprocessing characteristics of both RCZa and RCZp revealed a more caudal cingulate region $(8,10,40)$ with response-related activation, but no strong effects of feedback. However, this result was obtained in an explorative whole-brain fixed-effects analysis. Given the strong hypothesis-driven nature of this report, we prefer to limit the inferences of this study to those regions for which we had explicit hypotheses.

Overall, these results are consistent with a series of recent studies showing that portions of the general region labeled 'cingulate cortex' are involved in action selection based on the expected outcome of an action (Bush et al., 2002; Hadland et al., 2003; Shima and Tanji, 1998), integrating information regarding a motor response and its potential outcome (Williams et al., 2004). Our results illustrate how a specific portion of the medial frontal cortex, the RCZa, might receive evaluative information, which can be used to adapt behavior accordingly (Holroyd et al., 2004a; Ridderinkhof et al., 2004). Conceptually, our results are also consistent with the notion that ACC activation during error trials is the result of an error in reward prediction, indicating that ongoing events are unexpectedly disadvantageous and that this information is subsequently used to guide action selection (Holroyd and Coles, 2002).

In conclusion, in this study, we have illustrated the dynamic characteristics of the interplay between external and internal sources of error information, emphasizing the contribution of a specific portion of medial frontal cortex $(\mathrm{RCZa})$ to the selection of appropriate behaviors.

\section{Acknowledgments}

The authors would like to thank Paul Gaalman for excellent technical assistance and Peter Hagoort, Karl Magnus Petersson, and Guillen Fernandez for helpful comments on an earlier draft of the manuscript. C.B.H. was supported by National Institute of Mental Health (NIMH) postdoctoral fellowship MH63550. S.N. was supported by the Netherlands Organization for Scientific Research (NWO).

\section{References}

Akkal, D., Bioulac, B., Audin, J., Burbaud, P., 2002. Comparison of neuronal activity in the rostral supplementary and cingulate motor areas during a task with cognitive and motor demands. Eur. J. Neurosci. 15, $887-904$.

Bush, G., Luu, P., Posner, M.I., 2000. Cognitive and emotional influences in anterior cingulate cortex. Trends Cogn. Sci. 4, 215-222.

Bush, G., Vogt, B.A., Holmes, J., Jenike, M.A., Rosen, B.R., 2002. Dorsal anterior cingulate cortex: a role in reward-based decision making. Proc. Natl. Acad. Sci. U. S. A. 99, 523-528.

Carter, C.S., Braver, T.S., Barch, D.M., Botvinick, M.M., Noll, D., Cohen, J.D., 1998. Anterior cingulate cortex, error detection, and the online monitoring of performance. Science 280, 747-749.

Critchley, H.D., Mathias, C.J., Joseph, J.P., O’Doherty, J., Zanini, S., Dewar, B.K., Cipolotti, L., Shallice, T., Dolan, R.J., 2003. Human cingulate cortex and autonomic control: converging neuroimaging and clinical evidence. Brain 126, 2139-2152.
Dickinson, A., 1985. Actions and habits: the development of behavioural autonomy. Philos. Trans. R. Soc. London, B 308, 67-78.

Fiehler, K., Ullsperger, M., Von Cramon, D.Y., 2004. Neural correlates of error detection and error correction: is there a common neuroanatomical substrate? Eur. J. Neurosci. 19, 3081-3087.

Friston, K.J., Holmes, A.P., Worsley, K.J., Poline, J.P., Frith, C.D., Frackowiak, R.S.J., 1995. Statistical parametric maps in functional imaging: a general linear approach. Hum. Brain Mapp. 2, 189-210.

Friston, K.J., Fletcher, P., Josephs, O., Holmes, A.P., Rugg, M.D., Turner, R., 1998. Event-related fMRI: characterizing differential responses. NeuroImage 7, 30-40.

Friston, K.J., Zarahn, E., Josephs, O., Henson, R.N.A., Dale, A.M., 1999. Stochastic designs in event-related fMRI. NeuroImage 10, 607-619.

Garavan, H., Ross, T.J., Murphy, K., Roche, R.A.P., Stein, E.A., 2002. Dissociable executive functions in the dynamic control of behavior: inhibition, error detection, and correction. NeuroImage 17, 1820-1829.

Gehring, W.J., Goss, B., Coles, M.G.H., Meyer, D.E., Donchin, E., 1993. A neural system for error detection and compensation. Psychol. Sci. 4, $385-390$.

Genovese, C.R., Lazar, N.A., Nichols, T., 2002. Thresholding of statistical maps in functional neuroimaging using the false discovery rate. NeuroImage 15, 870-878.

Hadland, K.A., Rushworth, M.F.S., Gaffan, D., Passingham, R.E., 2003. The anterior cingulate and reward-guided selection for actions. J. Neurophysiol. 89, 1161-1164.

Holmes, A.P., Friston, K.J., 1998. Generalisability, random effects and population inference. NeuroImage 7, S754.

Holroyd, C.B., Coles, M.G.H., 2002. The neural basis of human error processing: reinforcement learning, dopamine, and the error-related negativity. Psychol. Rev. 109, 679-709.

Holroyd, C.B., Nieuwenhuis, S., Mars, R.B., Coles, M.G.H., 2004a. Anterior cingulate cortex, selection for action, and error processing. In: Posner, M.I. (Ed.), Cognitive Neuroscience of Attention. Guilford Press, New York, pp. 219-231.

Holroyd, C.B., Nieuwenhuis, S., Yeung, N., Nystrom, L., Mars, R.B., Coles, M.G.H., Cohen, J.D., 2004b. Dorsal anterior cingulate cortex shows fMRI response to internal and external error signals. Nat. Neurosci. 7, 498-974.

Holroyd, C.B., Yeung, N., Coles, M.G.H., Cohen, J.D., 2005. A mechanism for error detection in speeded response time tasks. J. Exp. Psychol. Gen. 134, 163-191.

Matsumoto, K., Tanaka, K., 2004. The role of the medial prefrontal cortex in achieving goals. Curr. Opin. Neurobiol. 14, 178-185.

Maxwell, S.E., Delany, H.D. (1990). Designing Experiments and Analyzing Data. Brooks/Cole Publishing. Pacific Grove.

Nachev, P., Rees, G., Parton, A., Kennard, C., Husain, M., 2005. Volition and conflict in human medial frontal cortex. Curr. Biol. 15, $122-128$.

Picard, N., Strick, P.L., 1996. Motor areas of the medial wall: a review of their location and functional activation. Cereb. Cortex 6 , $342-353$.

Picard, N., Strick, P.L., 2001. Imaging the premotor areas. Curr. Opin. Neurobiol. 11, 663-672.

Poline, J.B., Kherif, F., Penny, W., 2004. Contrasts and classical inference. In: Frackowiak, R.S.J. (Ed.), Human Brain Function, second ed. Academic Press, London, pp. 761-778.

Ridderinkhof, K.R., Ullsperger, M., Crone, E.A., Nieuwenhuis, S., 2004. The role of the medial frontal cortex in cognitive control. Science 306 , 443-447.

Rushworth, M.F.S., Walton, M.E., Kennerley, S.W., Bannerman, D.M., 2004. Action sets and decisions in the medial frontal cortex. Trends Cogn. Sci. 8, 410-417.

Shima, K., Tanji, J., 1998. Role for cingulate motor area cells in voluntary movement selection based on reward. Science 282, 1335-1338.

Shima, K., Mushiake, H., Saito, N., Tanji, J., 1996. Role for cells in the presupplementary motor area in updating motor plans. Proc. Natl. Acad. Sci. U. S. A. 93, 8694-8698. 
Talairach, J., Tournoux, P., 1988. Co-Planar Stereotaxic Atlas of the Human Brain. Thieme Medical Publishers, New York.

Thoenissen, D., Zilles, K., Toni, I., 2002. Differential involvement of parietal and precentral regions in movement preparation and motor intention. J. Neurosci. 22, 9024-9034.

Toni, I., Schluter, N.D., Joseph, J.P., Friston, K.J., Passingham, R.E., 1999. Signal-, set- and movement-related activity in the human brain: an event-related fMRI study. Cereb. Cortex 9, 35-49.

Toni, I., Ramnani, N., Josephs, O., Ashburner, J., Passingham, R.E., 2001. Learning arbitrary visuo-motor associations: temporal dynamic of brain activity. NeuroImage 14, 1048-1057.

Ullsperger, M., Von Cramon, D.Y., 2001. Subprocesses of performance monitoring: a dissociation of error processing and response competition revealed by event-related fMRI and ERPs. NeuroImage 14, $1387-1401$
Ullsperger, M., Von Cramon, D.Y., 2003. Error processing using external feedback: specific roles of the habenular complex, the reward system, and the cingulate motor area revealed by fMRI. J. Neurosci. 23, 4308-4314.

Ullsperger, M., Von Cramon, D.Y., 2004. Neuroimaging of performance monitoring: error detection and beyond. Cortex 40, 593-604.

Walton, M.E., Devlin, J.T., Rushworth, M.F.S., 2004. Interactions between decision making and performance monitoring within prefrontal cortex. Nat. Neurosci. 7, 1259-1265.

Williams, Z.N., Bush, G., Rauch, S.L., Cosgrove, G.R., Eskandar, E.N., 2004. Human anterior cingulate neurons and the integration of monetary reward with motor responses. Nat. Neurosci. 7, $1370-1375$.

Wise, S.P., Murray, E.A., 2000. Arbitrary associations between antecedents and actions. Trends Neurosci. 23, 271-276. 